\title{
IO'S ULTRAVIOLET SPOT EMISSION AS A PROBE OF THE JOVIAN MAGNETIC FIELD MODEL
}

\author{
V. Shaposhnikov*†, G. Litvinenko ${ }^{\ddagger}$, H. O. Rucker ${ }^{\S}$, V. Zaitsev*, \\ and A. Konovalenko
}

\begin{abstract}
The creation of the model of the Jovian magnetic field is the object of many papers. The measurements of the magnetic field in situ by Pioneer, Voyager, and Ulysses and remote observations of the positions of ultraviolet (UV) emission sources located in Jupiter's ionosphere along the trace of footprints of the Jovian satellites Io, Europe, and Ganymede were used for the modeling. This provided the modeling of the internal magnetic field in a region close to the planet quite accurate. However, these data is not sufficient for allowing an accurate modeling in a distance from the planet surface and from regions not sampled by spacecraft. In the present report we discuss the possibility to use the UV emissions from the sources located in the atmosphere of Io as a probe of the Jovian magnetic field near Io's orbit. We base it on the fact that the brightness of Io's UV emission observed from the compact sources (UV equatorial spots) at Io's atmosphere demonstrates strong correlation with the magnetic longitude of the moon. According to our model of the UV equatorial spots the brightness of the UV emission (FUV) from the spots is proportional to the forth degree of the value of the planetary magnetic field (B), $F U V \propto B 4$, along Io's obit. Comparing the modeled and observed UV brightness from the equatorial spots gives a possibility to refine the Jovian magnetic field model near Io's orbit.
\end{abstract}

\footnotetext{
* Institute of Applied Physics, Russian Academy of Sciences, Nizhny Novgorod, Russia

$\dagger$ National Research University High School of Economics, Nizhny Novgorod, Russia

$\ddagger$ Institute of Radio Astronomy, National Academy of Sciences of the Ukraine, Kharkov, Ukraine

$\S$ Commission for Astronomy, Austrian Academy of Sciences, Graz, Austria
} 
